\title{
Etiological and Epidemiological of Viral Diarrhea Among Children Under the Age of Five in Shenyang From 2018 to 2020
}

\section{Xinxin Dong}

Jilin University

\section{Ying Qi}

Shenyang Center for Disease Control and Prevention

\section{Ruiyu Chai}

Jilin University

\section{Han Xu}

Jilin University

\section{Jin Wang}

Jilin University

\section{Yingshuang Wang}

Jilin University

Ye Chen

Shenyang Center for Disease Control and Prevention

\section{Linlin Zhang}

Shenyang Center for Disease Control and Prevention

\section{Ying Lu}

Shenyang Center for Disease Control and Prevention

\section{Huijie Chen}

Shenyang Center for Disease Control and Prevention

yan yao ( $\nabla$ yaoyan@jlu.edu.cn )

Jilin University https://orcid.org/0000-0002-1823-3684

\section{Research Article}

Keywords: Diarrhea, Children, Etiology, Rotavirus, Sentinel surveillance

Posted Date: August 19th, 2021

DOI: https://doi.org/10.21203/rs.3.rs-817333/v1 
License: (c) (i) This work is licensed under a Creative Commons Attribution 4.0 International License. Read Full License 


\section{Abstract}

Diarrhea is one of the leading causes of death among children, especially in the age under five, but little data are available on the etiology of viral diarrhea in Shenyang. To understand the infection status and the relevant epidemiological characteristics of viral diarrhea and to fill gaps of how the distribution of viruses change across Shenyang in children under the age of five with diarrhea, stool specimens of children with diarrhea aged 0-59 months and surveillance data was collected from Sentinel Hospital of Shenyang. Rotavirus, calicivirus, adenovirus, and astrovirus 4 viruses were then analyzed, and the proportion of children who tested positive for each pathogen was calculated and seasonal and spatial patterns for major organisms were determined. Viruses were identified in $47.9 \%$ of the 897 samples from children with diarrhea. The main viruses of stool samples were rotavirus $(16.9 \%$, predominant type G9P[8]), calicivirus (14.7\%, norovirus, predominant type GII ), adenovirus (11.8\%), and astrovirus (4.5\%). Viral infections were mainly detected in the age of 0-12 months. In the area of Shenyang, Huanggu has the most cases $(198,22.1 \%)$, followed by Dadong $(137,15.3 \%)$ and Hunnan $(135,15.1 \%)$. The positive rate of viruses in patients of different ages, seasons, and regions was not same. Public health entities and the government should develop corresponding measures for different age groups, seasons, and regions.

\section{Introduction}

Diarrhea is one of the leading causes of death among children under the age of five and 525,00 children deaths annually from all over the world[1]. In China, infectious diarrhea is a category $C$ legal infectious disease, with an incidence of 2.50-3.38 episodes per person-year in children younger than five[2]. Diarrhea can be caused by bacterial, viral, and parasitic pathogens[3]. And the previous study has shown that even many pathogens can cause diarrhea, viruses account for over 75\%[4]. Viral diarrhea is mainly caused by rotavirus, calicivirus including norovirus and sapovirus, adenovirus and astrovirus[5, 6]. Also, the etiology of viral diarrhea differs between regions due to their local economic level[7], sanitary conditions[8], meteorological factors[9], and geography[10]. A better understanding of the epidemiology, etiology, and seasonality of viral diarrhea would be valuable for planning and adopting targeted preventive measures, as well as a clinical therapy. Shenyang,as one of the capital cities of Northeast China, few studies have evaluated viral agents known to be related to diarrhea and none explored the spatiotemporal patterns. Therefore, it is necessary to research viral diarrhea in Shenyang.

In this study, we were not only described the epidemiological features of Enteric viruses but also characterized rotavirus and norovirus strains circulating. We also filled gaps in how the distribution of viruses changes across Shenyang. Scientific pieces of evidence for early intervention and prevention can be provided by our results.

\section{Materials And Methods}

\section{Study area}


Shenyang is the capital city of Liaoning Province which is located at latitude $41^{\circ} 11^{\prime}-43^{\circ} 02^{\prime} \mathrm{N}$ and longitude $122^{\circ} 25^{\prime}-123^{\circ} 48^{\prime} \mathrm{E}$. It is a prefecture-level city in China, including urban and rural areas. It consists of 13 districts[11]. Among those 13 districts, Heping, Shenhe, Dadong, Huanggu, Tiexi, Sujiatun, Hunnan, Shenbeixin, Liaozhong, and Yuhong belong to the urban areas. Kangping, Faku, and Xinmin belong to rural areas.

\section{Study population and fecal specimens}

From 2018 to 2020, ongoing surveillance of diarrhea among children under the age of five was conducted in Sentinel hospitals of Shenyang. Inpatients of sentinel hospitals were registered, and a standard case definition was used to determine eligibility. Diarrhea[12] was defined as $\geq 3$ passages of watery, loose, mucus-, or bloody stools within a 24-h period. Stool specimens were collected by a sampler and quickly placed into a sterile sampling tube and stored in a biosafety transport box at $4-8^{\circ} \mathrm{C}$. They were sent to the laboratory of Shenyang Center for Disease Control and Prevention within 2 hours, frozen at $-80^{\circ} \mathrm{C}$, and tested in batches. Also, the relevant demographic and epidemiological data were collected.

\section{Virus detection}

Four enteric viruses including rotavirus, calicivirus, adenovirus, and astrovirus were detected. The isolation and identification of enteric virus were performed using the unified standards operation laid down by the Chinese Center for Disease Control and Prevention[12]. The methods are briefly described below.

For rotavirus: stool samples were analyzed for rotavirus by using an enzyme-linked immunosorbent assay (ELISA) kit (ProSpecT Rotavirus kit, Oxoid Ltd, Basingstoke, UK). The operation process according to the instructions of the manufacturer. Then RNA of the ELISA-positive samples was extracted (Geneaid Biotech, Taiwan, China) and carried out with reverse transcription-polymerase chain reactions (RT-PCR) for the determination of $G$ and $P$ genotypes.

For calicivirus, adenovirus, and astrovirus: those viruses were detected by real-time RT-PCR. The Real-time RT-PCR products positive for samples of calicivirus were analyzed (One-Step RT-PCR Kit, Qiangen, Germany) for the determination of genotypes.

\section{Data analysis}

The SPSS 24.0 was used to perform statistical analysis on the collected data. The Chi-square test and Fisher's exact test were used to test for statistical significance. The P-value of less than 0.05 was considered significant. ArcGIS 10.3 was used to explore the spatiotemporal patterns of viral diarrhea.

\section{Results}

\section{Characteristics of case-patients}


During the data collection, a total of 897 hospitalized children aged 0-59 months (mean age $22 \pm 16$ months) were recruited in this investigation, of which 541 (60.3\%) were male, and $356(39.7 \%)$ were female. Among them, 322 individuals (35.9\%) aged 0-12 months, 221 (24.6\%) aged 13-24 months, 150 (16.7\%) aged 25-36 months, 120 (13.4\%) aged 37-48 months, and 84 (9.4\%) aged 49-59 months. Besides diarrhea, vomiting $(368,41.6 \%)$ was the most common clinical manifestation of cases presenting with concomitant vomiting. Other common symptoms included respiratory symptoms (177, 19.7\%), fever (172, $19.2 \%)$, and nervous system symptoms $(7,0.8 \%)$. Of the 897 stool specimens submitted to be detected, watery stool $(732,81.6 \%)$ was the most common type, followed by mucus stool $(85,9.5 \%)$. In the area of Shenyang, Huanggu had the largest number of cases $(198,22.0 \%)$, followed by Dadong $(137,15.3 \%)$, hunnan $(135,15.1 \%)$, Yuhong $(98,10.9 \%)$, and Shenhe $(83,9.3 \%)$ (Table 1$)$.

Table 1 Demographic and epidemiological characteristics of patients with diarrhea 
Characteristic

No. of case (\%) Enteric virus infection

$$
(n=897) \quad(n=430)
$$

\section{Gender}

\begin{tabular}{|c|c|c|}
\hline Male & $541(60.3)$ & $269(62.6)$ \\
\hline Female & 356 (39.7) & $161(37.4)$ \\
\hline \multicolumn{3}{|l|}{ Seasons } \\
\hline Spring & $102(11.4)$ & $43(10.0)$ \\
\hline Summer & $142(15.8)$ & $33(7.7)$ \\
\hline Autumn & $296(33.0)$ & $141(32.8)$ \\
\hline Winter & 357 (39.8) & $213(49.5)$ \\
\hline \multicolumn{3}{|c|}{ Age (months) } \\
\hline $0-12$ & 322 (35.9) & $129(30.0)$ \\
\hline $13-24$ & $221(24.6)$ & $121(28.1)$ \\
\hline $25-36$ & $150(16.7)$ & 87 (20.2) \\
\hline $37-48$ & $120(13.4)$ & $62(14.4)$ \\
\hline $49-59$ & $84(9.4)$ & $31(7.2)$ \\
\hline \multicolumn{3}{|l|}{ Year } \\
\hline 2018 & 297 (33.1) & 203 (47.2) \\
\hline 2019 & 299 (33.3) & $119(27.7)$ \\
\hline 2020 & 301 (33.6) & $108(25.1)$ \\
\hline \multicolumn{3}{|l|}{ Region } \\
\hline Huanggu & $198(22.1)$ & $86(20.0)$ \\
\hline Dadong & $137(15.3)$ & $67(15.6)$ \\
\hline hunnan & $135(15.1)$ & 74 (17.2) \\
\hline Yuhong & $98(10.9)$ & $50(11.6)$ \\
\hline Shenhe & $83(9.3)$ & $40(9.3)$ \\
\hline Tiexi & $80(8.9)$ & $36(8.4)$ \\
\hline Sujiatun & $42(4.7)$ & $19(4.4)$ \\
\hline Heping & $39(4.3)$ & $14(3.3)$ \\
\hline
\end{tabular}




\begin{tabular}{lll} 
Shenbeixin & $35(3.9)$ & $21(4.9)$ \\
\hline Xinmin & $24(2.7)$ & $13(3.0)$ \\
\hline Liaozhong & $12(1.3)$ & $7(1.6)$ \\
\hline Faku & $10(1.1)$ & $3(0.7)$ \\
\hline Kangping & $4(0.4)$ & $0(0.0)$ \\
\hline Diarrhea & & \\
\hline Watery & $732(81.6)$ & $357(83.0)$ \\
\hline Mucus & $85(9.5)$ & $40(9.3)$ \\
\hline Rice-water & $8(0.9)$ & $5(1.2)$ \\
\hline Bloody & $2(0.2)$ & $1(0.2)$ \\
\hline Blood purulent & $1(0.1)$ & $0(0.0)$ \\
\hline Other symptoms & & \\
\hline Vomiting & $368(41.6)$ & $212(49.3)$ \\
\hline Respiratory & $177(19.7)$ & $82(19.1)$ \\
\hline Fever & $172(19.2)$ & $80(18.6)$ \\
\hline Nervous symptoms & $7(0.8)$ & $2(0.5)$
\end{tabular}

\section{Prevalence of viruses}

Enteric viral infections were found in 430 of the 897 cases $(47.9 \%, 430 / 897)$, and rotavirus was the most commonly identified $(16.9 \%, 152 / 897)$, followed by calicivirus $(14.7 \%, 132 / 897)$, adenovirus $(11.8 \%$, 106/897), and astrovirus $(4.5 \%, 40 / 897)$. Among calicivirus, 98 cases were norovirus. Of the mixed infection cases, eight children were infected by two viruses, and rotavirus mixed one of the other three enteric pathogens was the most common pattern (Table 2).

Table 2 Viral pathogens identified in hospitalized children in Shenyang 


\begin{tabular}{ll} 
Virus & No. (\%) of stool specimens \\
\hline Rotavirus & $152(16.9)$ \\
\hline Calicivirus & $132(14.7)$ \\
(Norovirus) & $(98(10.9))$ \\
\hline Adenovirus & $106(11.8)$ \\
\hline Astrovirus & $40(4.5)$ \\
\hline Calicivirus + Adenovirus & $1(0.1)$ \\
\hline Adenovirus + Astrovirus & $2(0.2)$ \\
\hline Rotavirus+ Astrovirus & $2(0.2)$ \\
\hline Rotavirus + Calicivirus & $1(0.1)$ \\
\hline Rotavirus + Adenovirus & $1(0.1)$ \\
\hline Calicivirus + Astrovirus & $1(0.1)$
\end{tabular}

There was no significant difference in the detection rate of rotavirus, calicivirus, adenovirus and astrovirus in children of different genders $\left(c^{2}=1.776, P=0.183 ; c^{2}=0.426, P=0.513 ; c^{2}=0.384, P=0.536 ; c^{2}=0.384, P=0.535\right)$. And the prevalence of diarrhea viruses among age groups was compared. Viral agents were more likely to be identified in the group of 0-12 ( $n=129)$ and 13-24 ( $n=121)$ months. Also, with aging, the number of cases decreased gradually. The distribution of those four viruses was varied among children of different age groups in different regions (Fig. 1). Infection cases were found in almost all age groups in Hunnan, Huanggu, Dadong, and Yuhong, and other urban areas of Shenyang. Among the four viruses, only the positive rate of adenovirus was statistically significant in different month age groups $\left(c^{2}=21.914, P \otimes 0.001\right)$.

The isolation rates of rotavirus, calicivirus, adenovirus and astrovirus four enteric viral pathogens showed a similar distinct seasonal variation with a higher rate in winter and autumn (Fig. 2a). The seasonal curve had a peak in winter and a trough in summer. And there was a significant difference in rotavirus, calicivirus, adenovirus, and astrovirus between seasons $\left(\mathrm{c}^{2}=40.020, P \otimes 0.001 ; \mathrm{c}^{2}=49.759, P\right]$ $\left.0.001 ; c^{2}=25.395, P \otimes 0.001 ; c^{2}=16.335, P=0.001\right)$. Similarly, from Fig. $2 c$, the virus infection between different districts in Shenyang also had a seasonal trend, and the infection situation in each district was different. During the high incidence period of viral diarrhea, the number of cases of viruses was higher in Dadong, Huanggu, Hunnan, Shenhe, and Yuhong five districts.

From 2018 to 2020, the number of cases of rotavirus, adenovirus, and astrovirus had a clear downtrend. But with the passage of time, the trend of calicivirus was different from the others (Fig. 2b). And there was a significant difference in rotavirus, calicivirus, adenovirus, and astrovirus between years $\left(c^{2}=59.486, P \otimes 0.001 ; c^{2}=25.782, P \otimes 0.001 ; c^{2}=51.146, P \otimes 0.001 ; c^{2}=21.105, P \otimes 0.001\right)$. 
In our study, a strong regional pattern in rotavirus, calicivirus, adenovirus, and astrovirus four viruses was also observed (Fig. 3). Huanggu, Dadong, hunnan, and Yuhong four districts had a relatively high positive rate of viruses compared to other areas in Shenyang. And there had a significant difference in rotavirus, adenovirus, and astrovirus between different areas of Shenyang $\left(c^{2}=30.977, P=0.001 ; c^{2}=23.017, P=0.018 ; c^{2}=32.911, P \otimes 0.001\right)$.

\section{Genotypes of rotavirus and norovirus}

Among 152 genotyped rotavirus strains, G9P[8], G2P[4], G3P[8], G9P[4], and G9P[6] were the leading genotypes identified, accounting for $84.5 \%, 3.9 \%, 3.1 \%, 1.6 \%$, and $0.8 \%$, respectively. Among caliciviruses, 98 cases of norovirus were detected, and the Gll was the predominant genogroup of norovirus, accounting for $85.7 \%$ of positive samples, followed by GI (11,11.2\%), and GI+GII (3, 3.1\%). During the three-year study period, strains of rotavirus and norovirus were circulated in children changed from year to year. The prevalence of G9P[8] was increased from $83 \%$ to $100 \%$ since 2018 and became the only virus strain in 2020. In 2018 and 2019, the only genotype of norovirus was GIl, while the main genotypes in 2020 were GI, GII, and GI+GII (Figure 4).

From Fig. 5, we could conclude that in each district of Shenyang, the distribution of predominant genotypes of rotavirus (G9P[8]) and norovirus (GII) were different. For G9P[8], it was mainly concentrated in Huanggu (26, 23.9\%), Shenhe (20, 18.3\%), Hunnan (19, 17.4\%), and Yuhong (16, 14.7\%). And GII genotype was relatively high in Dadong $(15,17.9 \%)$, followed by Huanggu $(13,15.5 \%)$, Yuhong $(12$, $14.3 \%)$, and Hunnan (12, 14.3\%).

\section{Discussion}

In this study, the updated results on the viral etiology as well as epidemiological characteristics of children under the age of five with viral diarrhea were obtained by studying their stool samples in sentinel hospitals in Shenyang from 2018 to 2020 . These etiologies differ in terms of the demographic characteristics of the infected children, the season and year of prevalence, and the region.

During the period of this study, 897 children aged 0-59 months who were hospitalized in Shenyang Sentinel Hospital provided stool samples, of which $47.9 \%$ were virus-positive. Rotavirus and calicivirus were the predominant viral agents. This result was similar to previous studies[9, 13]. It's worth noting that the number of cases of rotavirus had a downtrend but the calicivirus had an upward trend. Although no data were available on rotavirus vaccination rates among children in Shenyang, with the coverage of the rotavirus vaccine, the number of diarrhea deaths attributable to rotavirus had already declined and a subsequent increase in calicivirus (norovirus)-associated viral diarrhea was observed[14]. Suggesting that we should pay more attention to the impact of calicivirus on infants and young children. The prevention and control measures of calicivirus (norovirus) should be developed. Now there are some antiviral medicines, disinfectants, and vaccines[15]. And the inclusion of a rotavirus vaccine in the national immunization program is still strongly recommended. Adenovirus and astrovirus infections were $11.8 \%$ 
and $4.5 \%$, respectively. Although the roles of these two viruses in viral diarrhea were minor, they should not be neglected because they still affect the health of children and the global burden of disease[1, 16].

Epidemic strains were varied over the place in both developed and developing countries[17]. In our study, we also found a similar conclusion. The predominant strain of rotavirus was G9P[8], increasing from 83\% to $100 \%$ from 2018 to 2020 . For norovirus, the genotype of norovirus includes only GII among 2018 and 2019, but in 2020 included GI, GII, and GI+GII three types. In addition, the G9P[8] and GII were almost concentrated in urban such as Huanggu, Hunnan, and Yuhong. Suggesting that it is essential to conduct continuous surveillance in different areas of Shenyang, especially in urban areas, to understand the prevalence of this viral diarrhea caused by G9P[8] and GII strains.

The infection rate of the male was higher than female. This may be because boys had a higher chance of being exposed to unsanitary conditions. Although there was no statistically significant difference in gender, it's also suggested that boys should pay more attention to personal hygiene. Also, the distribution of the virus in different age groups was explored. In general, the frequency and types of viral infections were higher in young children, especially in 0-12 and 13-24 months. With the increase in age, the number of cases decreased gradually. This difference in age distribution may be influenced by the host immunity, eating habits, hygiene, and environment[18, 19]. In China, kindergarten education is at the age of 3-5 years, possibly leading to the establishment of the host protective immunity and the ensuing decreased infection after this age. In addition, most of the children over 2 years old have been vaccinated or have been infected or their immune systems are well developed. Protective antibodies are produced in the body and are not prone to infectious diarrhea. Above all, under the age of two groups are the most vulnerable group to viral diarrhea, they should be taken as primary active surveillance targets.

The analysis of the epidemiological trends of viral diarrhea revealed that the infection rate of viral diarrhea decreased year by year from 2018 to 2020. This change may be related to the number of patients consulted, the hygiene habits of children, and their nutritional status. In terms of seasonal distribution, viral diarrhea can occur throughout the year, and its peak epidemic is mainly concentrated in autumn and winter, which is consistent with the results reported in related studies[20]. We hypothesized that the potential reason for this not only includes the characteristics of the virus (suitable for cold and dry environments) but also in spring and winter, infants are more preference for indoor activities. With doors and windows closed, children can easily inhale viruses from contaminated environments. Shenyang is located in the northern part of China, with cold weather and long winters, suggesting that we should pay attention to seasonal protection.

Due to local economic conditions, sanitary conditions, and other factors, the incidence of viral diarrhea may be different between different cities[1]. In our study, we also found that even in the same city, infection rates may be varied in different regions. An obvious spatial distribution in viral diarrhea could be seen in Shenyang. In particular, it was more prevalent in Huanggu, Dadong, Hunnan, Yuhong, Shenhe, and Tiexi five districts. Other regions were relatively lower. These five districts are the urban areas of Shenyang. The result was similar to a previous study in Beijing[17]. These districts are the economic, 
cultural, transportation, and commercial centers[11]. Relatively high population density and population mobility, which may lead to poor sanitation and make children more vulnerable to exposure to viruses. Or the results may be due to parents of these kids have different medical awareness in other regions, so mild infectious diarrhea may not seek medical care and remain unreported, leading to biases in the incidence of the disease.

In conclusion, we studied the viral pathogens spectrum and relevant epidemiology of viral diarrhea in Sentinel Hospital of Shenyang, to provide evidence for the prevention and treatment of viral diarrhea. We demonstrate that there is a high incidence of viral diarrhea, particularly due to rotavirus infections in Shenyang. And the etiologies differ in terms of the demographic characteristics of the infected children, the season and year of prevalence, and region. This suggests that we should take targeted measures for different situations, the relevant departments should pay more attention to viral diarrhea in infants and young children in both basic research and clinical diagnosis and treatment, conduct long-term systematic surveillance, and carry out relevant health education activities. Providing a scientific basis for the prevention, control, and immunization of viral diarrhea in infants and young children in China. Besides, the rotavirus vaccine in the national immunization program is strongly necessary.

\section{Limitations}

This study had several limitations. First, the number of cases was not as many as others reported in multicenter studies due to restrictions on time and resources. Second, children with mild infectious diarrhea may not seek medical care and remain unreported, leading to biases in the incidence of the disease. Nevertheless, this study will enrich the epidemiology database of China and will be of great importance for preventing and control viral diarrhea.

\section{Declarations}

\section{Acknowledgments}

We acknowledge the staff in the Shenyang CDC and surveillance hospitals, who collected demographical data and stool specimens.

\section{Conflict of interests}

No conflict of interest exits in the submission of this manuscript, and manuscript is approved by all authors for publication.

\section{Ethics and consent to participate}

This study was approved by the ethical review committee at the Shenyang Center for Disease Control and Prevention (Shenyang CDC, Shenyang, China). Each fecal specimen was collected with the informed consent of the children's parents. 


\section{Funding sources}

None

\section{Author contributions}

All authors participated in interpreting the findings. Xinxin Dong and Ruiyu Chai designed the study and drafted the manuscript. Ying QI, Ye Chen, Linlin Zhang, Ying Lu, and Huijie Chen were responsible for sample and surveillance data collection. Xinxin Dong, Jin Wang, Yingshuang Wang, and Han Xu were involved in the analysis and processing of the data. All authors read and approved the final manuscript.

\section{References}

1. Collaborators GBDDD. Quantifying risks and interventions that have affected the burden of diarrhoea among children younger than 5 years: an analysis of the Global Burden of Disease Study 2017. Lancet Infect Dis. 2020;20:37-59. https://doi.org/10.1016/S1473-3099(19)30401-3

2. Chen J, Wan CM, Gong ST, Fang F, Sun M, Qian Y, et al. Chinese clinical practice guidelines for acute infectious diarrhea in children. World J Pediatr. 2018;14:429-36. https://doi.org/10.1007/s12519018-0190-2

3. Liu X, Meng L, Li J, Liu X, Bai Y, Yu D, et al. Etiological epidemiology of viral diarrhea on the basis of sentinel surveillance in children younger than 5 years in Gansu, northwest China, 2009-2013. J Med Virol. 2015;87:2048-53. https://doi.org/10.1002/jmv.24283

4. Ren Z, Kong Y, Wang J, Wang Q, Huang A, Xu H. Etiological study of enteric viruses and the genetic diversity of norovirus, sapovirus, adenovirus, and astrovirus in children with diarrhea in Chongqing, China. BMC Infect Dis. 2013;13:412. https://doi.org/10.1186/1471-2334-13-412

5. Zheng S, Yu F, Chen X, Cui D, Cheng Y, Xie G, et al. Enteropathogens in children less than 5 years of age with acute diarrhea: a 5-year surveillance study in the Southeast Coast of China. BMC Infect Dis. 2016;16:434. https://doi.org/10.1186/s12879-016-1760-3

6. Ouyang $\mathrm{Y}, \mathrm{Ma} \mathrm{H}$, Jin $\mathrm{M}$, Wang $\mathrm{X}$, Wang $\mathrm{J}$, Xu L, et al. Etiology and epidemiology of viral diarrhea in children under the age of five hospitalized in Tianjin, China. Arch Virol. 2012;157:881-7. https://doi.org/10.1007/s00705-012-1235-9

7. Breurec S, Vanel N, Bata P, Chartier L, Farra A, Favennec L, et al. Etiology and Epidemiology of Diarrhea in Hospitalized Children from Low Income Country: A Matched Case-Control Study in Central African Republic. PLoS Negl Trop Dis. 2016;10:e0004283. https://doi.org/10.1371/journal.pntd.0004283

8. Islam M, Rahman M, Unicomb L, Kafi MAH, Rahman M, Alam M, et al. Child defecation and feces management practices in rural Bangladesh: Associations with fecal contamination, observed hand cleanliness and child diarrhea. PLoS One. 2020;15:e0236163. https://doi.org/10.1371/journal.pone.0236163 
9. Fang X, Ai J, Liu W, Ji H, Zhang X, Peng Z, et al. Epidemiology of infectious diarrhoea and the relationship with etiological and meteorological factors in Jiangsu Province, China. Sci Rep. 2019;9:19571. https://doi.org/10.1038/s41598-019-56207-2

10. Collaborators GBDDD. Estimates of the global, regional, and national morbidity, mortality, and aetiologies of diarrhoea in 195 countries: a systematic analysis for the Global Burden of Disease Study 2016. Lancet Infect Dis. 2018;18:1211-28. https://doi.org/10.1016/S1473-3099(18)30362-1

11. JU SYSTJ. shenyang yearbook. In: JU SYSTJ, editor. Beijing2020.

12. Prevention NloVDCa. Viral Diarrhea Surveillance Program. Beijing: National Institute of Viral Disease Control and Prevention; 2017.

13. Chang H, Guo J, Wei Z, Huang Z, Wang C, Qiu Y, et al. Aetiology of acute diarrhoea in children in Shanghai, 2015-2018. PLoS One. 2021;16:e0249888. https://doi.org/10.1371/journal.pone.0249888

14. Farahmand M, Moghoofei M, Dorost A, Shoja Z, Ghorbani S, Kiani SJ, et al. Global prevalence and genotype distribution of norovirus infection in children with gastroenteritis: A meta-analysis on 6 years of research from 2015 to 2020. Rev Med Virol. 2021:e2237. https://doi.org/10.1002/rmv.2237

15. Ramani S, Atmar RL, Estes MK. Epidemiology of human noroviruses and updates on vaccine development. Curr Opin Gastroenterol. 2014;30:25-33. https://doi.org/10.1097/MOG.0000000000000022

16. Banyai K, Estes MK, Martella V, Parashar UD. Viral gastroenteritis. Lancet. 2018;392:175-86. https://doi.org/10.1016/S0140-6736(18)31128-0

17. Tian Y, Chughtai AA, Gao Z, Yan H, Chen Y, Liu B, et al. Prevalence and genotypes of group A rotavirus among outpatient children under five years old with diarrhea in Beijing, China, 2011-2016. BMC Infect Dis. 2018;18:497. https://doi.org/10.1186/s12879-018-3411-3

18. Ethelberg S, Olesen B, Neimann J, Schiellerup P, Helms M, Jensen C, et al. Risk factors for diarrhea among children in an industrialized country. Epidemiology. 2006;17:24-30. https://doi.org/10.1097/01.ede.0000187621.41373.0a

19. Baker KK, O'Reilly CE, Levine MM, Kotloff KL, Nataro JP, Ayers TL, et al. Sanitation and HygieneSpecific Risk Factors for Moderate-to-Severe Diarrhea in Young Children in the Global Enteric Multicenter Study, 2007-2011: Case-Control Study. PLoS Med. 2016;13:e1002010. https://doi.org/10.1371/journal.pmed.1002010

20. Yu J, Jing H, Lai S, Xu W, Li M, Wu J, et al. Etiology of diarrhea among children under the age five in China: Results from a five-year surveillance. J Infect. 2015;71:19-27. https://doi.org/10.1016/j.jinf.2015.03.001

\section{Figures}


a

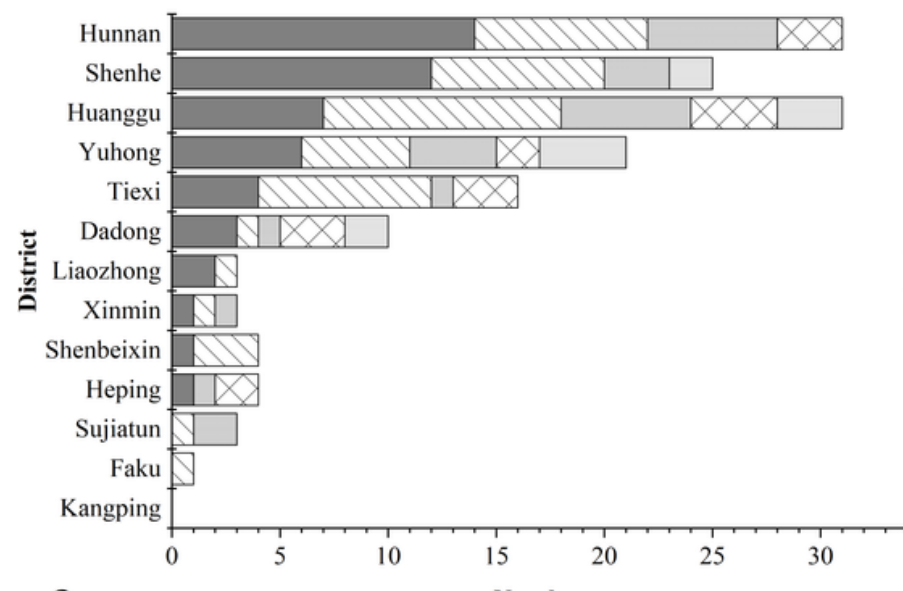

C

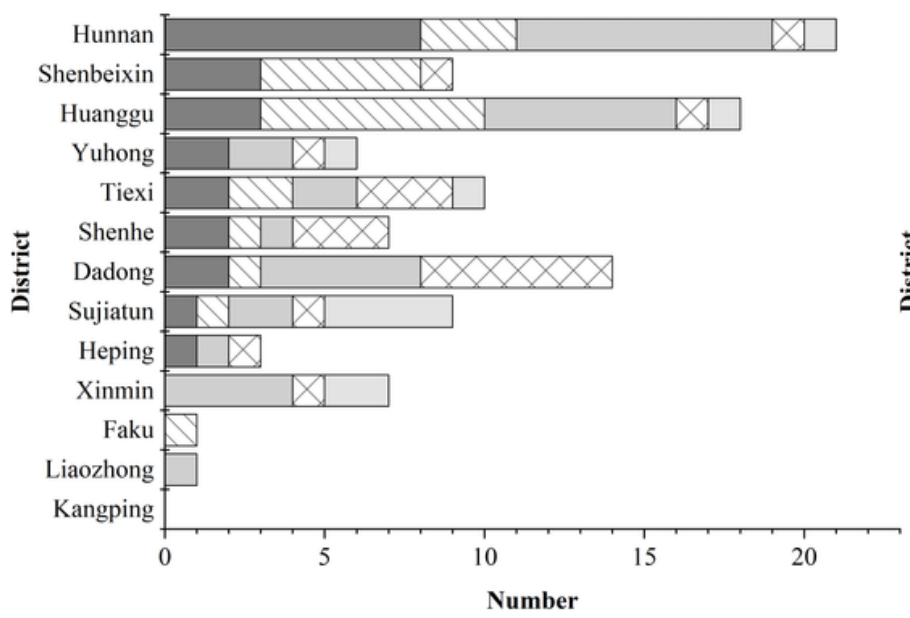

$\mathrm{b}$

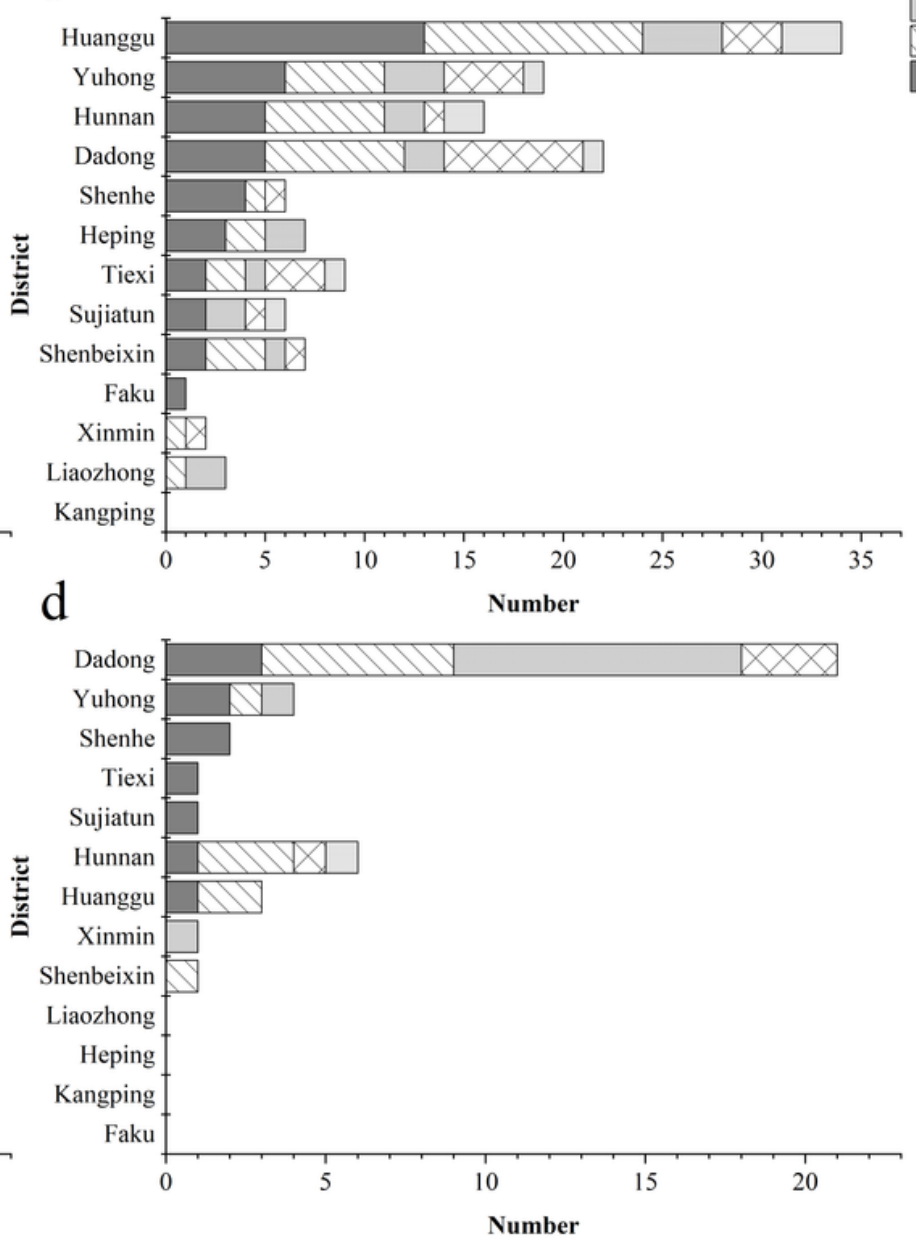

\section{Figure 1}

Distribution of different viral infections by age groups in different regions

a

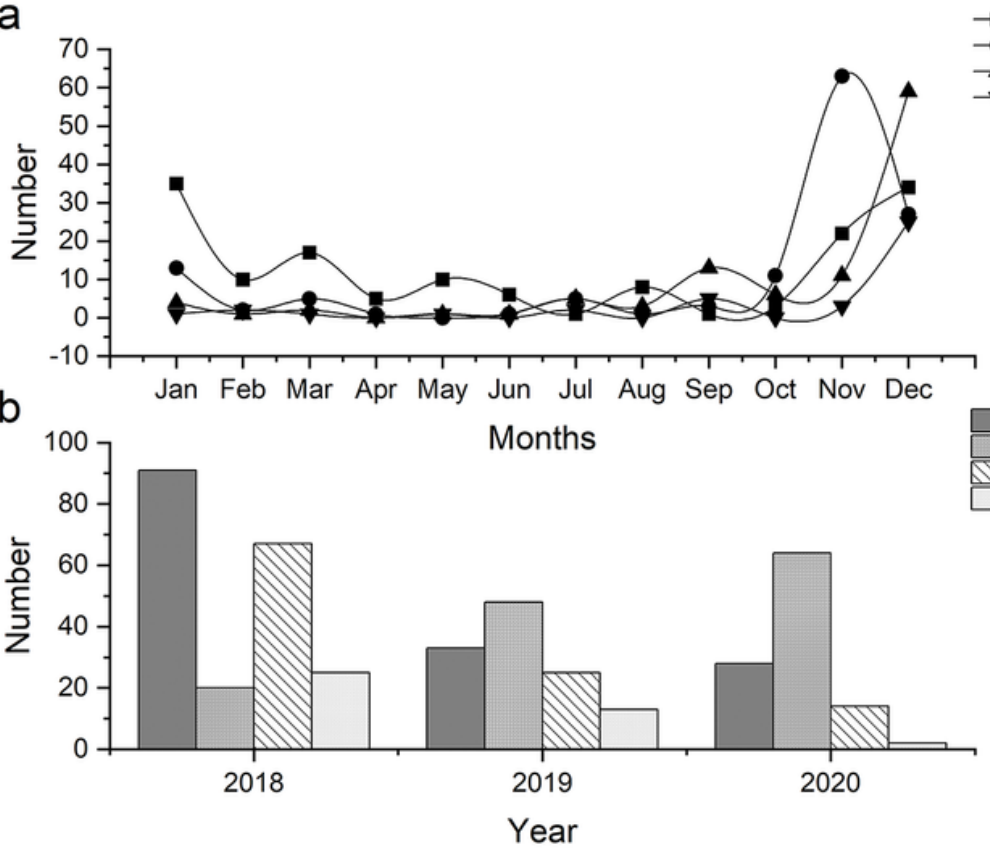

C

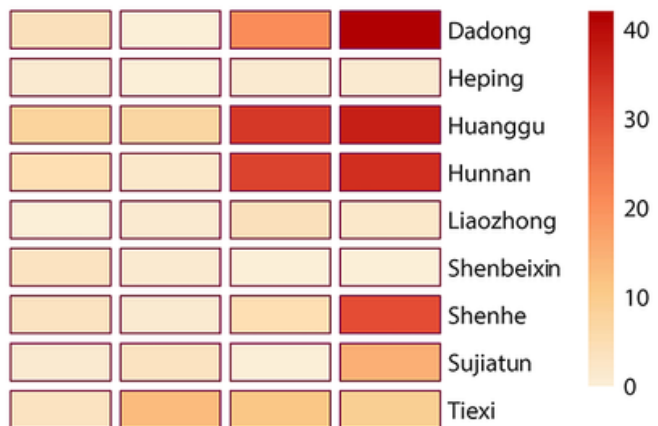


Figure 2

Distribution of different viral infections by (a) month, (b) year, (c) regions
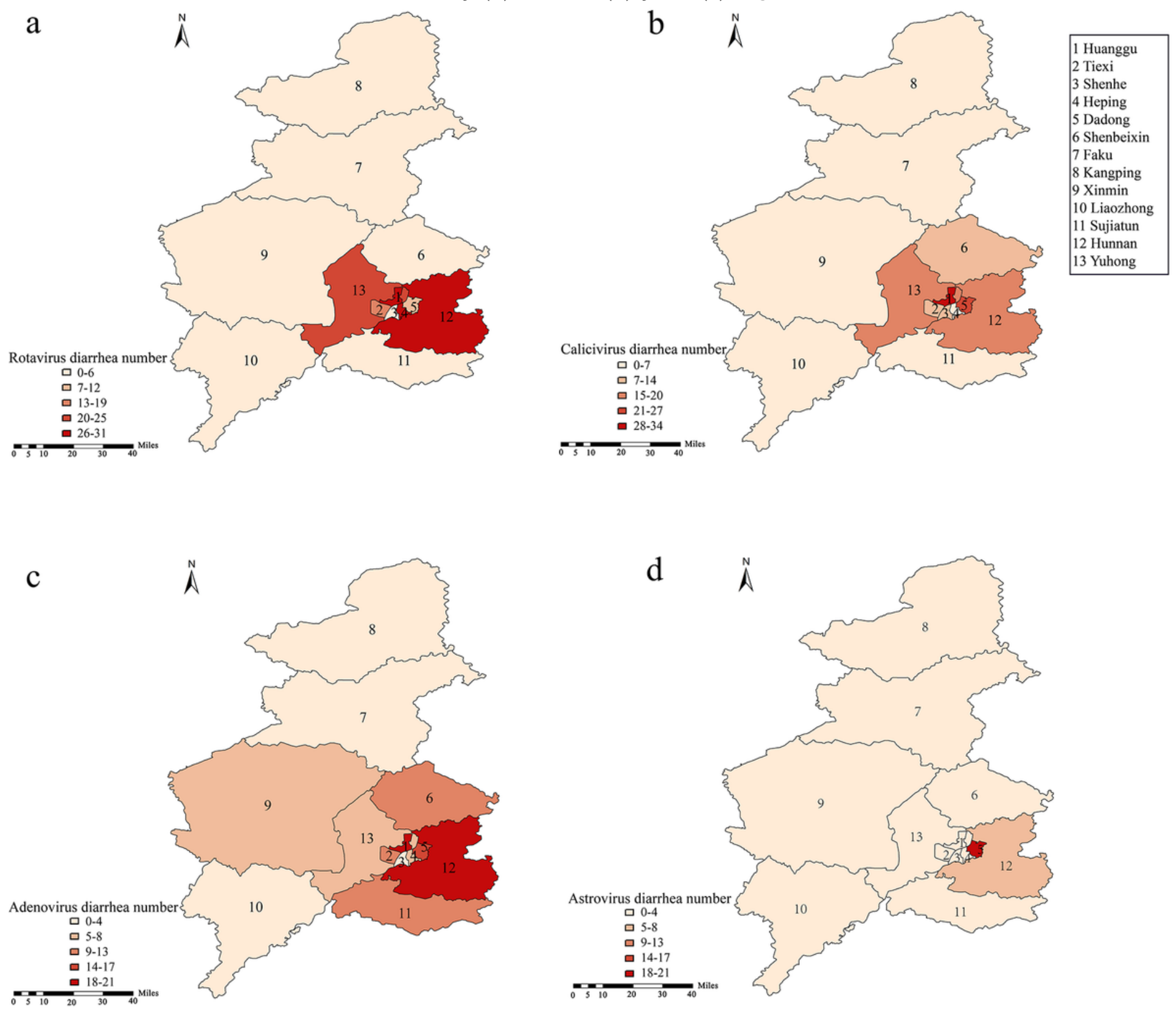

Figure 3

Distribution for (a) rotavirus, (b) calicivirus, (c) adenovirus, (d) astrovirus among different regions of Shenyang 


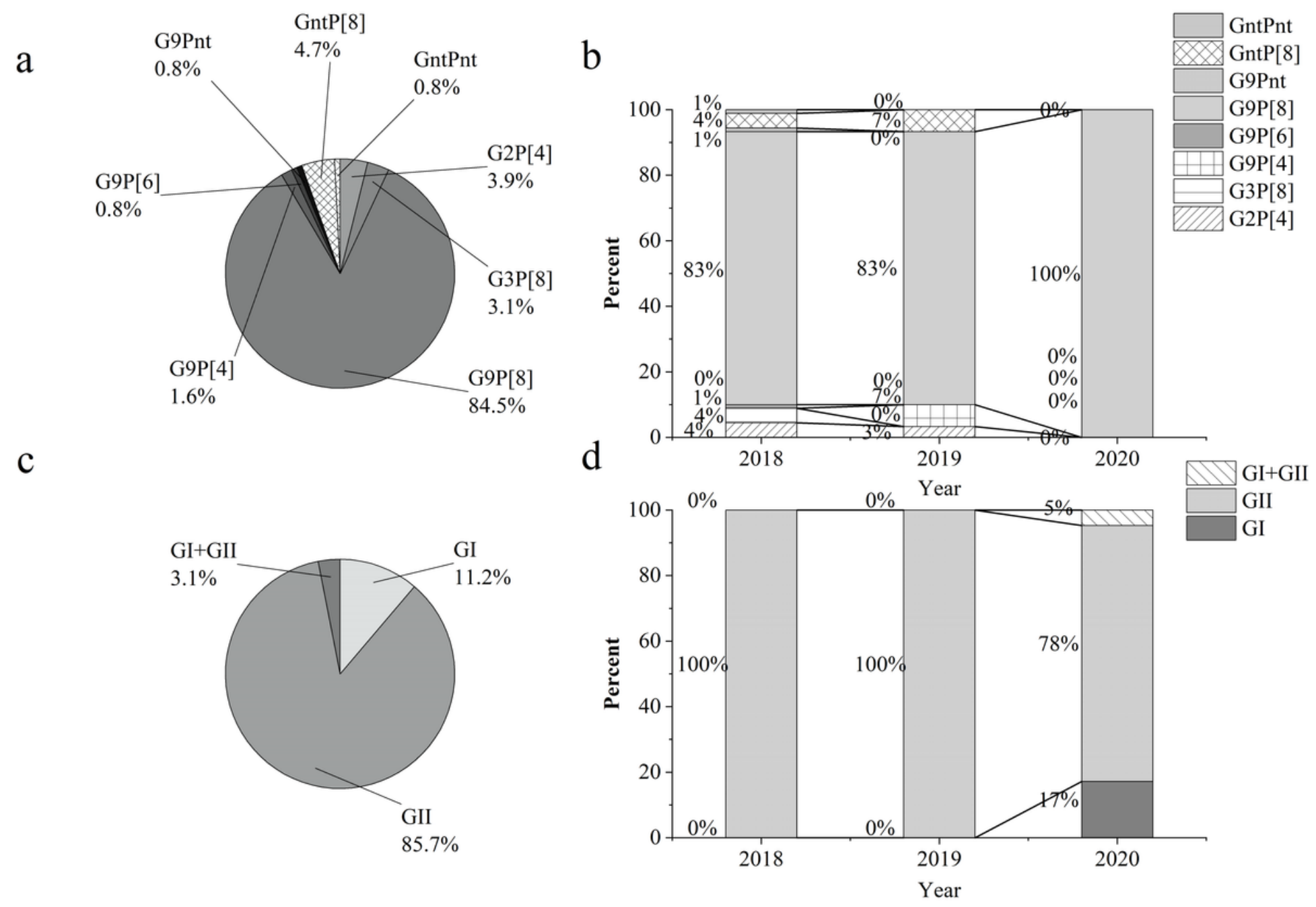

Figure 4

Distribution of rotavirus(a-b) and norovirus (c-d) genotypes from 2018 to 2020
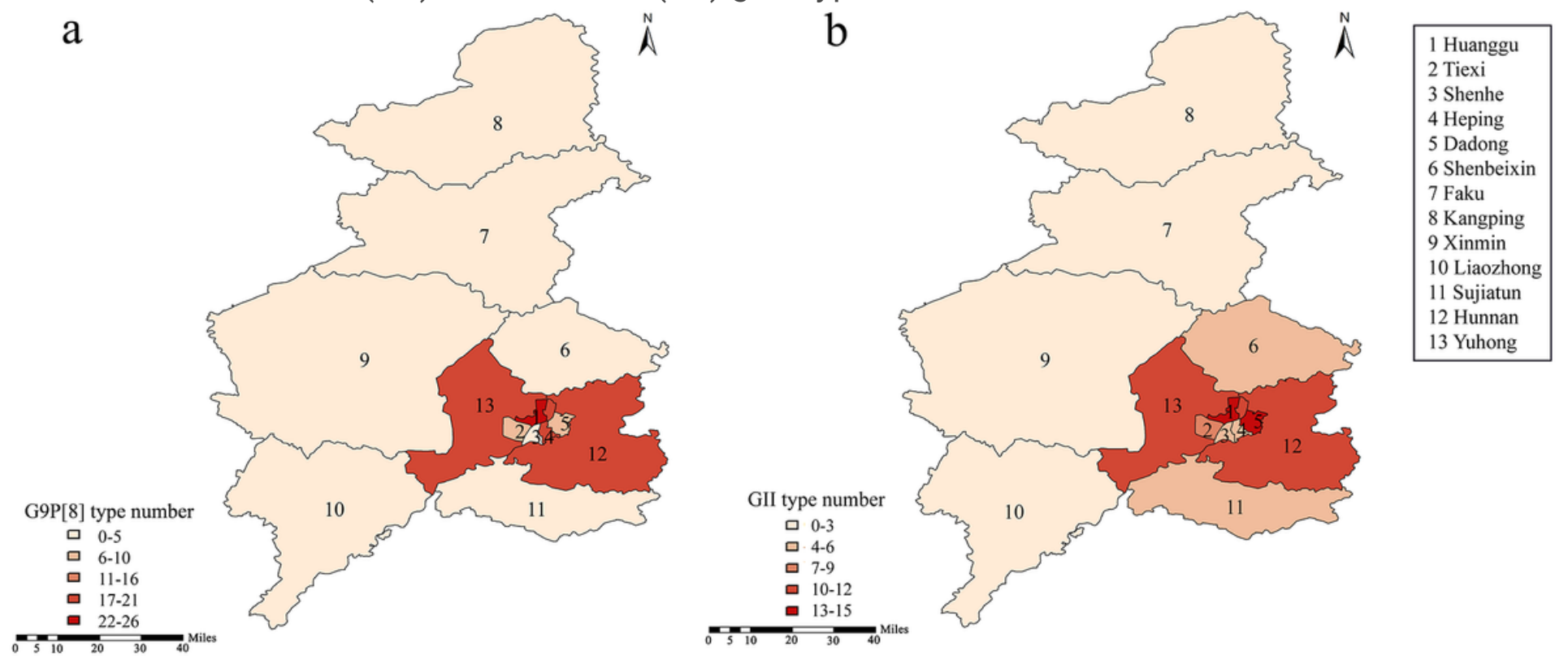

Figure 5 
Distribution for (a) G9[P]8, (b) GIl in different regions of Shenyang 